\title{
Nutritional Status and Associated Factors Among Children Below Five Years of Age in Somali Region, Ethiopia: Evidence From 2016 Ethiopian Demographic and Health Survey
}

Damitie Kebede ( $\square$ dakebede10@gmail.com )

College of Agriculture and Environmental Sciences, P O Box, 5501, Bahir Dar University, Bahir Dar, Ethiopia https://orcid.org/0000-0001-5494-9906

Hayat Aragaw

Bahir Dar University

Eyerusalem Worku

Bahir Dar University

Research

Keywords: Under-five children, Underweight, Wasting, Stunting, Ethiopia

Posted Date: September 9th, 2020

DOI: https://doi.org/10.21203/rs.3.rs-69254/v1

License: (c) (1) This work is licensed under a Creative Commons Attribution 4.0 International License.

Read Full License 


\section{Abstract}

Background: Childhood under-nutrition is far-reaching in low and middle-income nations. Under-nutrition is one of the major open wellbeing concerns among newborn children and youthful children in Ethiopia. This study aimed to assess the prevalence of under-nutrition and its related variables among under-five children in Somali Region, Ethiopia.

Methods: The data for this study was extricated from the Ethiopian Demographic and Health Survey (EDHS) 2016. The data collected from 1339 children born 5 years before was considered within the analysis. A multivariable binary logistic regression analysis was utilized at a $5 \%$ level of significance to decide the individual and community-level variables related to childhood malnutrition.

Results: the incidences of stunting, underweight and wasting were $27.4 \%, 28.7 \%$ and $22.7 \%$, respectively. About $16.1 \%$ of children were both stunted and underweight; the extent of both being underweight and wasted was $11.7 \%$, the prevalence of both stunted and wasted children was $5.5 \%$, and all three malnutrition conditions were $4.7 \%$ children. Among the variables considered in this study, age of the child in months, type of birth, anemia level, size child at birth, sex of the child, mothers' BMI and sources of drinking water were significantly related to stunting, underweight and wasting in Somali Region.

Conclusions: The prevalence of stunting, underweight and wasting was relatively high. Under-nutrition is one of the major open wellbeing concerns among children in Somali region. The impact of these variables ought to be considered to develop strategies for decreasing the lack of healthy sustenance due to malnutrition in the study areas. Hence, intercession should be centered on making strides the undernutrition determinant variables of the children to be solid, to improve the child's wholesome status, and decrease child mortality quickly.

\section{Introduction}

Globally, approximately 151 million children under five years of age suffer from chronic malnutrition and 67 million under-five were wasted in 2017 [1]. Lack of healthy sustenance is of open wellbeing significance in creating nations and is dependable for over half of child passing each year from preventable causes [2]. Among this malnourished under-five, $55 \%$ of all stunted children under-five years of age have been from Asia and $39 \%$ were living in Africa, whereas $69 \%$ of all wasted children under-five years of age dwelled in Asia and $27 \%$ of wasted under-five lived in Africa [3].

Malnutrition among under-five declined from 32.6-22.2\% between 2000 and 2017 around the world [4]. In 2018, UNICEF detailed that around 3.1 million children passed on of under-nutrition and malnutrition contributes to more than half of worldwide child deaths [5]. Although there's a global decay within the malnutrition rates of under-5, the chance of lack of healthy sustenance remains tall and it is the major cause of under-five morbidity and mortality of African particularly sub-Saharan Africa [6]. Childhood under-nutrition is broad in low and middle-income nations. In these nations, it is an imperative and backhanded cause of child mortality. Around the world, stunting and wasting beside intrauterine 
development limitations are mindful of about 2.1 million deaths in under-five children that contain $21 \%$ of all deaths [7].

Ethiopia has illustrated promising advances in decreasing levels of under-nutrition over the past two decades. Be that as it may, under-nutrition is one of the major open wellbeing concerns among newborn children and youthful children within the nation $[8,9]$. The pattern levels of under-nutrition remain high that the nation still has to continue considerable speculation in nourishment [10]. In 2016, about 155 million children beneath the age of years were enduring from stunting. Under-nutrition contributes about $45 \%$ of deaths among children beneath the age of 5 years [11]. All-inclusive a million children were underweight, 51 million were stunted and 17 million children were wasted in 2013 Besides, under-nutrition is credited with lacking feeding; defective feeding practices hones rehashed contaminations like diarrheal infections, acute respiratory infections and worm pervasions [12-14]. Under-five children are the foremost vulnerable age group for under-nutrition especially the under-nutrition in creating nation like Ethiopia. The issue of under-nutrition within the early stages of life can increment chance infections, morbidity, and mortality alongside diminished mental and cognitive advancement [15].

Child under-nutrition and mortality rates are sensible reactions to both wellbeing programs and financial conditions such as pay, unemployment, and lack of education [16]. In Ethiopia, the predominance of under-nutrition is high [17-21] which ranges from $14.6 \%$ in Addis Ababa to $46.3 \%$ in Amhara region for stunting, 3.5\% in Addis Ababa to 22.5\% in Somalia region for wasting and 5\% in Addis Ababa to 35.5\% in Afar region for underweight [22]. The country has begun executing health extension packages in which nourishment is one of the most components included in the health extension package.

Even though studies have been carried out on the recognizable proof of components that are related to children under five years old of malnutrition in the Somali region, none of them employments the national representative data for the Somali region. The exertion made in decreasing under-five children's malnutrition in the region is still high, and more effort is required to move forward the obstructions for advance diminishment. More research studies are, subsequently, required to inform policymakers to execute suitable mediation programs. To address these gaps, an all-inclusive cross-sectional analysis of the recent 2016 Ethiopian Demographic Health Survey (EDHS) was done, to survey the prevalence and investigate the major risk factors of malnutrition among under-five children in Somali region, Ethiopia.

\section{Methods}

\section{Description of study design and area}

Cross-sectional study design was used for this study. The study was conducted in Somali regional state which is located in the east and southeast of Ethiopia. According to the 2007 Census, the state's population birth weight was $4,439,147$ of which 1,970,363 were males. The urban residents of the region were 621,210 and its rural residents $3,817,937$ [23].

\section{Inclusion and exclusion variables}


Mothers/caregivers who had young children under five years old who live in the Somali regional state for at least 6 months were included in the study and those who had mental illnesses interfering with the interview were not considered in the study.

\section{Data Sources}

The data on this study was extricated from the EDHS 2016. The 2016 EDHS utilized two-stage stratified sampling to choose households. In the first stage, there were 645 enumeration areas (202 in urban and 443 in rural areas) based on the 2007 Ethiopia Population and Housing Census (PHC). In the second stage, A total of 18,008 households were considered, of which 16,650 ( $98 \%$ of response rate) households were eligible. The women were interviewed by distributing questioners and information on their birth history [24]. Of 1339 (700 males and 639 females) under-five children from Somali region were considered for this consider.

\section{Variables of the study}

The subordinate factors for this consideration were the malnutrition status of under-5 year children (stunting, underweight and wasting). Children whose height-for-age Z-score is below minus two standard deviations (-2SD) from the median of the reference population is considered as stunted. If the weight-forage Z-score is below minus two standard deviations (-2 SD) from the medium of reference population then the child is underweight. Children whose weight for height Z-score is below minus two standard deviations (-2SD) from the median of the reference population are considered as wasted [25]. Illustrative factors were chosen after conducting a point by point writing survey [25-30] additionally accessible with complete data in the EDHS, 2016 data set was included within the current investigation. Selected illustrative factors were divided into two levels which included socio-demographic-maternal and childlevel variables. Socio demographic-maternal variables chosen were types of residence, household wealth index, mother's educational level, mother's body mass list (BMI), religion, and type of toilet facility, sex of household head and Sources of drinking water. Child-level components were the sex of the child, child age, type of birth, Number of living children and child size at birth.

\section{Statistical data analysis}

The data was extricated, altered, and analyzed by using SPSS form 23 for Windows. The descriptive statistics such as frequencies and proportions were used to summarize the dissemination of chosen foundation characteristics of the sample. To assess the impact of each socio-demographic-maternal and child-level components on under-five stunting, wasting, and underweight (odds ratio (OR) with 95\% confidence intervals $(\mathrm{Cl})$, logistic regression analysis was fitted. Bivariate logistic regression was performed and a variable with a P-value of less than 0.25 was transported into a multivariable binary logistic regression analysis to identify the determinant of malnutrition of under-five children. Finally, variables with P-values $<0.05$ in the multivariable logistic regression model was taken as statistically significant. 


\section{Results}

In this study, a total of 1339 under-five children were included. In the Afar region, the incidences of stunting, underweight and wasting were $27.4 \%, 28.7 \%$ and $22.7 \%$, respectively. Almost $16.1 \%$ of children were both stunted and underweight; the proportion of underweight and wasting was $11.7 \%$, the proportion of stunted and wasted was $5.5 \%$, and all three malnutrition conditions were only $4.7 \%$ children.

Among the participants, 555 children (41.4\%) were 0-24 months old, 501 (37.4\%) were $25-47$ months old and $283(21.1 \%)$ were $48-59$ months old. The male children constituted 700 of the sample population (52.3\%), and the females constituted 639 (47.7\%). Nearly 1069 (79.8\%) of the children were taken from rural areas while the, remaining $270(20.2 \%)$ of children were taken from urban areas. More than half of the children (62.4\%) were anemic and the rest of the children (37.6\%) were non-anemic. The most children were born in single birth type (98.2\%), and only $1.8 \%$ of children were born multiple birth type. The majority of children (56.5\%) were from overweight mothers, $24.6 \%$ of children were from normal-weight mothers and only $18.8 \%$ of children were from underweight mothers. Regarding the educational status of the household, $85.4 \%$ of households did not attend at all, $10.7 \%$ of household attended primary school and $3.8 \%$ of households attended secondary and higher education. The majority (63.8\%) of the children were born at normal size, $32.3 \%$ were in large size and $24.6 \%$ of the children were born in small size at birth. Similarly, more than half $(63.8 \%)$ of the respondents had no toilet facilities, and $36.2 \% \%$ had toilet facilities. Nearly one child in five children (81.9\%) was from the household who used unimproved water while the remaining $18.1 \%$ of children were from the household who used improved water sources. The majority of children (75.8\%) were born from poor wealth index families, $18.4 \%$ of children were from rich wealth index family and the rest $5.8 \%$ of children were from medium wealth index families. The number of children who lived in household members of $1-2,3-4$ and $>4$ was $20.9 \%, 30.4 \%$ and $48.7 \%$, respectively. More than half of the respondents were male household headed and the rest $32.3 \%$ of the respondents were female household headed.

\section{Factors Associated With Stunting}

According to the multivariable logistic regression analysis, age of a child, type of birth, toilet facility and anemia level were significantly associated with being stunted. The risk of being stunted was 1.94 and 1.66 times more likely among children that were aged $25-47$ and $48-59$ as compared to those aged 024 months, respectively. Male children were 0.23 times less likely to be stunted as compared to female children. The odds of stunting among children who were born in multi birth types were 2.86 times higher as compared to those born in single birth type. Children from the household who had not toilet facility were 1.45 times more likely to be stunted as compared to the children household who had a toilet facility. Children born from the medium and rich households were 0.44 and 0.22 times less likely to develop stunting as compared to those born from poor households. Being stunted of anemic children was 2.36 times more likely to be stunted as compared to non-anemic children (Table 1). 
Table 1

Bivariate and multivariable logistic regression of determinants associated with stunting on childhood under 5 years old in Somali Region, Ethiopia, EDHS 2016

\begin{tabular}{|c|c|c|c|c|}
\hline \multirow[t]{2}{*}{ Variables } & & Stunting & $\operatorname{COR}(95 \% \mathrm{Cl})$ & AOR $(95 \% \mathrm{Cl})$ \\
\hline & Yes & \multicolumn{3}{|l|}{ No } \\
\hline \multicolumn{5}{|c|}{ Age of child in months } \\
\hline $0-24$ & $\begin{array}{l}115 \\
(20.7 \%)\end{array}$ & 440 (79.3\%) & 1 & 1 \\
\hline $25-47$ & $\begin{array}{l}172 \\
(34.4 \%)\end{array}$ & $328(65.6 \%)$ & $\begin{array}{l}2.01(1.52, \\
2.64)\end{array}$ & $\begin{array}{l}1.94(1.41 \\
2.69)^{\star \star \star}\end{array}$ \\
\hline $48-59$ & $80(28.3 \%)$ & $203(71.7 \%)$ & $\begin{array}{l}1.51(1.08, \\
2.10)\end{array}$ & $1.66(1.12,2.46)^{x}$ \\
\hline \multicolumn{5}{|c|}{ Sex of child } \\
\hline Male & $\begin{array}{l}202 \\
(28.9 \%)\end{array}$ & $497(71.1 \%)$ & 1 & 1 \\
\hline Female & $\begin{array}{l}165 \\
(25.8 \%)\end{array}$ & $474(74.2 \%)$ & $\begin{array}{l}1.17(0.92, \\
1.49)\end{array}$ & $0.77(0.58,1.02)$ \\
\hline
\end{tabular}

Place of residence

Urban

$69(25.7 \%)$

$200(74.3 \%) \quad 1$

1

Rural

298

(27.9\%)

$771(72.1 \%)$

$1.12(0.83$,

1.52)

$0.73(0.45,1.19)$

Type of birth

Single birth

357

(27.2\%)

$957(72.8 \%) \quad 1$

1

Multiple birth

$10(41.7 \%)$

$14(58.3 \%)$

$1.92(0.84$, 4.35)

$2.86(1.10,7.37)$ *

Mothers` BMI

Over weight

55 (21.8\%)

197

(780.2\%)

Normal weight

213

(28.2\%)

$543(71.8 \%)$

Under weight

$99(30.0 \%)$

$231(70.0 \%)$ 2.25)

1.41 (1.01,

1.97)

1.54 (1.05,

1

1

$1.07(0.73,1.58)$

$1.24(0.80,1.93)$

Size of child at birth

AOR: adjusted odds ratio; COR: crude odds ratio; $\mathrm{Cl}$ : confidence interval; * significant $\mathrm{p}$ value $<0.05$; ** significant $p$ value $<0.01 ; * *$ significant $p$ value $<0.001,1$ = reference 


\begin{tabular}{|c|c|c|c|c|}
\hline Variables & & Stunting & COR $(95 \% \mathrm{Cl})$ & AOR (95\% Cl) \\
\hline Large & $\begin{array}{l}105 \\
(24.2 \%)\end{array}$ & $328(75.8 \%)$ & 1 & 1 \\
\hline Medium & $\begin{array}{l}162 \\
(28.1 \%)\end{array}$ & $414(71.9 \%)$ & $\begin{array}{l}1.22(0.92, \\
1.63)\end{array}$ & $1.17(0.85,1.62)$ \\
\hline Small & $\begin{array}{l}100 \\
(30.4 \%)\end{array}$ & $229(69.6 \%)$ & $\begin{array}{l}1.36(0.99 \\
1.88)\end{array}$ & $1.24(0.86,1.79)$ \\
\hline \multicolumn{5}{|c|}{ Present of toilet facility } \\
\hline Yes & $\begin{array}{l}111 \\
(22.9 \%)\end{array}$ & $374(77.1 \%)$ & 1 & 1 \\
\hline No & $\begin{array}{l}256 \\
(30.0 \%)\end{array}$ & $597(70.0 \%)$ & $\begin{array}{l}1.73(0.51 \\
1.03)\end{array}$ & $\begin{array}{l}1.45(1.12 \\
1.87)^{\star \star}\end{array}$ \\
\hline \multicolumn{5}{|c|}{ Sex of household head } \\
\hline Male & $\begin{array}{l}259 \\
(28.6 \%)\end{array}$ & $648(71.4 \%)$ & 1 & 1 \\
\hline Female & $\begin{array}{l}108 \\
(25.1 \%)\end{array}$ & $323(74.9 \%)$ & $\begin{array}{l}0.84(0.64 \\
1.09)\end{array}$ & $0.95(0.71,1.28)$ \\
\hline \multicolumn{5}{|c|}{$\begin{array}{l}\text { Household wealth index } \\
\text { combined }\end{array}$} \\
\hline Poor & $\begin{array}{l}298 \\
(29.4 \%)\end{array}$ & $717(70.6 \%)$ & 1 & 1 \\
\hline Medium & $14(17.9 \%)$ & $64(82.1 \%)$ & $\begin{array}{l}0.53(0.29 \\
0.95)\end{array}$ & $0.56(0.28,1.11)$ \\
\hline Rich & $55(22.4 \%)$ & $190(77.6 \%)$ & $\begin{array}{l}0.70(0.50 \\
0.97)\end{array}$ & $0.78(0.43,1.40)$ \\
\hline \multicolumn{5}{|c|}{ Number of living children } \\
\hline $1-2$ & $70(25.1 \%)$ & $209(74.9 \%)$ & 1 & 1 \\
\hline $3-4$ & $\begin{array}{l}109 \\
(26.8 \%)\end{array}$ & $298(73.2 \%)$ & $\begin{array}{l}1.09(0.77 \\
1.55)\end{array}$ & $1.03(0.68,1.56)$ \\
\hline$>4$ & $\begin{array}{l}188 \\
(28.8 \%)\end{array}$ & $464(71.2 \%)$ & $\begin{array}{l}1.21(0.88 \\
1.67)\end{array}$ & $1.01(0.68,1.49)$ \\
\hline \multicolumn{5}{|c|}{ Sources of drinking water } \\
\hline Improved & $62(25.7 \%)$ & $179(74.3 \%)$ & 1 & 1 \\
\hline
\end{tabular}

AOR: adjusted odds ratio; COR: crude odds ratio; $\mathrm{Cl}$ : confidence interval; * significant $\mathrm{p}$ value $<0.05$; ** significant $p$ value $<0.01 ; * * *$ significant $p$ value $<0.001,1=$ reference 


\begin{tabular}{|c|c|c|c|c|}
\hline Variables & & Stunting & $\operatorname{COR}(95 \% \mathrm{Cl})$ & AOR (95\% Cl) \\
\hline Unimproved & $\begin{array}{l}305 \\
(27.8 \%)\end{array}$ & 792 (72.2\%) & $\begin{array}{l}1.11(0.81 \\
1.53)\end{array}$ & $0.82(0.55,1.23)$ \\
\hline \multicolumn{5}{|l|}{ Anemia level } \\
\hline Non-anemic & 34 (18.6\%) & 149 (81.4\%) & 1 & 1 \\
\hline Anemic & $\begin{array}{l}284 \\
(34.0 \%)\end{array}$ & $551(66.0 \%)$ & $\begin{array}{l}2.26(1.52, \\
3.37)\end{array}$ & $\begin{array}{l}2.36(1.55 \\
3.61)^{\star \star \star}\end{array}$ \\
\hline
\end{tabular}

\section{Factors Associated With Under-weight}

Results of the multivariable binary logistic regression model revealed that the age of the child, size of the child at birth, and anemia level were significantly associated with underweight. The children who were 25-47 and 48-59 aged groups were 1.67 and 1.70 times more likely to develop underweight as compared to those who were 0-24 aged groups. Compared to children large size at birth, the odds of underweight among children in the medium and small size at birth were 1.17 and 1.55 times higher. The odds of being wasting were 0.11 times lower among female children than male children. The anemic children were 1.65 times more likely to be underweight as compared to non-anemic children (Table 2). 
Table 2

Bivariate and multivariable logistic regression of risk factors associated with under-weight on childhood less than 5 years in Somali Region, Ethiopia, EDHS 2016

\begin{tabular}{|c|c|c|c|c|}
\hline \multirow[t]{2}{*}{ Variables } & & Underweight & $\operatorname{COR}(95 \% \mathrm{Cl})$ & AOR $(95 \% \mathrm{Cl})$ \\
\hline & Yes & \multicolumn{3}{|l|}{ No } \\
\hline \multicolumn{5}{|c|}{ Age of child in months } \\
\hline $0-24$ & $\begin{array}{l}124 \\
(22.3 \%)\end{array}$ & $431(77.7 \%)$ & 1 & 1 \\
\hline $25-47$ & $\begin{array}{l}171 \\
(34.1 \%)\end{array}$ & $330(65.9 \%)$ & $\begin{array}{l}1.80(1.37 \\
2.36)\end{array}$ & $\begin{array}{l}1.67(1.22 \\
2.28)^{\star \star \star}\end{array}$ \\
\hline $48-59$ & $89(31.4 \%)$ & $194(68.6 \%)$ & $\begin{array}{l}1.60(1.16 \\
2.20)\end{array}$ & $\begin{array}{l}1.70(1.17 \\
2.46)^{\star \star}\end{array}$ \\
\hline \multicolumn{5}{|c|}{ Sex of child } \\
\hline Male & $\begin{array}{l}204 \\
(29.1 \%)\end{array}$ & $496(70.9 \%)$ & 1 & 1 \\
\hline Female & $\begin{array}{l}180 \\
(28.2 \%)\end{array}$ & $459(71.8 \%)$ & $\begin{array}{l}0.95(0.76 \\
1.21)\end{array}$ & $0.89(0.68,1.16)$ \\
\hline
\end{tabular}

Place of residence

Urban

$73(27.0 \%)$

$197(73.0 \%) \quad 1$

1

Rural

311

$(29.1 \%)$

758 (70.9\%)

$1.11(0.82$,

1.49)

Type of birth

Single birth

376

(28.6\%)

Multiple birth

$8(33.3 \%)$

$16(66.7 \%)$

$1.25(0.53$,

2.94)

$939(71.4 \%) \quad 1$

1

$0.72(0.45,1.17)$

Mothers` BMI

Over weight

Normal weight

60 (23.8\%)

$192(76.2 \%)$

1

1

215

(28.4\%)

542 (71.6\%)

1.27 (0.91,

$1.10(0.75,1.61)$

Under weight

109

(33.0\%)

$221(67.0 \%)$

1.77)

$1.26(0.48,3.33)$

Size of child at birth

AOR: adjusted odds ratio; COR: crude odds ratio; $\mathrm{Cl}$ : confidence interval; * significant $\mathrm{p}$-value $<0.05$; ** significant $p$-value $<0.01$; significant $p$-value $<0.001,1=$ reference 


\begin{tabular}{|c|c|c|c|c|}
\hline Variables & & Underweight & $\operatorname{COR}(95 \% \mathrm{Cl})$ & AOR $(95 \% \mathrm{Cl})$ \\
\hline Large & $\begin{array}{l}109 \\
(25.2 \%)\end{array}$ & $324(74.8 \%)$ & 1 & 1 \\
\hline Medium & $\begin{array}{l}160 \\
(27.7 \%)\end{array}$ & 417 (72.3\%) & $\begin{array}{l}1.14(0.86 \\
1.51)\end{array}$ & $1.17(0.85,1.61)$ \\
\hline Small & $\begin{array}{l}115 \\
(35.0 \%)\end{array}$ & $214(65.0 \%)$ & $\begin{array}{l}1.60(1.71 \\
2.19)\end{array}$ & $1.55(1.08,2.21)^{*}$ \\
\hline \multicolumn{5}{|c|}{ Present of toilet facility } \\
\hline Yes & $\begin{array}{l}259 \\
(30.3 \%)\end{array}$ & $595(69.7 \%)$ & 1 & 1 \\
\hline No & $\begin{array}{l}125 \\
(25.8 \%)\end{array}$ & $360(74.2 \%)$ & $\begin{array}{l}1.25(0.98 \\
1.61)\end{array}$ & $0.84(0.60,1.18)$ \\
\hline \multicolumn{5}{|c|}{ Sex of household head } \\
\hline Male & $\begin{array}{l}265 \\
(29.2 \%)\end{array}$ & $642(70.8 \%)$ & 1 & 1 \\
\hline Female & $\begin{array}{l}119 \\
(27.5 \%)\end{array}$ & $313(72.5 \%)$ & $\begin{array}{l}0.92 \text { (0.71, } \\
1.19)\end{array}$ & $0.98(0.73,1.31)$ \\
\hline \multicolumn{5}{|c|}{$\begin{array}{l}\text { Household wealth index } \\
\text { combined }\end{array}$} \\
\hline Poor & $\begin{array}{l}302 \\
(29.8 \%)\end{array}$ & $713(70.2 \%)$ & 1 & 1 \\
\hline Medium & $24(30.8 \%)$ & $54(69.2 \%)$ & $\begin{array}{l}1.05(0.64 \\
1.73)\end{array}$ & $0.96(0.53,1.74)$ \\
\hline Rich & $58(23.6 \%)$ & $188(76.4 \%)$ & $\begin{array}{l}0.73(0.53 \\
1.01)\end{array}$ & $0.59(0.33,1.06)$ \\
\hline \multicolumn{5}{|c|}{ Number of living children } \\
\hline $1-2$ & $73(26.1 \%)$ & 207 (73.9\%) & 1 & 1 \\
\hline $3-4$ & $\begin{array}{l}122 \\
(30.0 \%)\end{array}$ & $285(70.0 \%)$ & $\begin{array}{l}1.21(0.86 \\
1.71)\end{array}$ & $1.16(0.78,1.75)$ \\
\hline$>4$ & $\begin{array}{l}189 \\
(29.0 \%)\end{array}$ & $463(71.0 \%)$ & $\begin{array}{l}1.16(0.84 \\
1.59)\end{array}$ & $1.11(0.75,1.63)$ \\
\hline \multicolumn{5}{|c|}{ Sources of drinking water } \\
\hline Improved & $69(28.5 \%)$ & 173 (71.5\%) & 1 & 1 \\
\hline
\end{tabular}

AOR: adjusted odds ratio; COR: crude odds ratio; $\mathrm{Cl}$ : confidence interval; * significant $\mathrm{p}$-value $<0.05$; ** significant $p$-value $<0.01$; significant $p$-value $<0.001,1=$ reference 


\begin{tabular}{|c|c|c|c|c|}
\hline Variables & & Underweight & COR $(95 \% \mathrm{Cl})$ & AOR (95\% Cl) \\
\hline Unimproved & $\begin{array}{l}315 \\
(28.7 \%)\end{array}$ & 782 (71.3\%) & $\begin{array}{l}1.01(0.74 \\
1.37)\end{array}$ & $0.89(0.61,1.32)$ \\
\hline \multicolumn{5}{|l|}{ Anemia level } \\
\hline Non-anemic & $46(25.0 \%)$ & $280(70.9 \%)$ & 1 & 1 \\
\hline Anemic & $\begin{array}{l}287 \\
(34.4 \%)\end{array}$ & $548(65.6 \%)$ & $\begin{array}{l}1.57(1.09 \\
2.26)\end{array}$ & $1.65(1.12,2.43) *$ \\
\hline
\end{tabular}

\section{Factors Associated With Wasting}

Based on multivariable logistic regression analysis, sex of the child, Mothers's BMI, size of child at birth and sources of drinking water were significantly associated with wasting. The results of the adjusted showed that male children were 0.35 times less likely to be wasting compared to female children. The risk of being wasting among children who were born from underweight and normal-weight mothers were 1.64 and 1.38 times higher compared to those born from overweight mothers. The children who had small size at birth were 1.58 times more likely to be wasting compared to those who had large size at birth. Children whose households used unimproved water were 1.66 times more likely to be wasting as compared to the children's household used improved water (Table 3 ). 
Table 3

Bivariate and multivariate logistic regression of risk factors associated with wasting on childhood less than 5 years old in Somali Region, Ethiopia, EDHS 2016.

\begin{tabular}{|c|c|c|c|c|}
\hline \multirow[t]{2}{*}{ Variables } & & Wasting & $\operatorname{COR}(95 \% \mathrm{Cl})$ & AOR $(95 \% \mathrm{Cl})$ \\
\hline & Yes & \multicolumn{3}{|l|}{ No } \\
\hline \multicolumn{5}{|c|}{ Age of child in months } \\
\hline $0-24$ & $\begin{array}{l}120 \\
(21.6 \%)\end{array}$ & $435(78.4 \%)$ & 1 & 1 \\
\hline $25-47$ & $\begin{array}{l}117 \\
(23.4 \%)\end{array}$ & $384(76.6 \%)$ & $\begin{array}{l}1.10(0.83 \\
1.48)\end{array}$ & $1.07(0.76,1.49)$ \\
\hline $48-59$ & $67(23.7 \%)$ & $216(76.3 \%)$ & $\begin{array}{l}1.12(0.80 \\
1.58)\end{array}$ & $1.14(0.76,1.72)$ \\
\hline \multicolumn{5}{|l|}{ Sex of child } \\
\hline Male & $\begin{array}{l}181 \\
(25.9 \%)\end{array}$ & $519(74.1 \%)$ & 1 & 1 \\
\hline Female & $\begin{array}{l}123 \\
(19.2 \%)\end{array}$ & $516(80.8 \%)$ & $\begin{array}{l}0.68(0.53 \\
0.89)\end{array}$ & $\begin{array}{l}0.65(0.48 \\
0.88)^{\star \star}\end{array}$ \\
\hline \multicolumn{5}{|c|}{ Place of residence } \\
\hline Urban & $65(24.1 \%)$ & $205(75.9 \%)$ & 1 & 1 \\
\hline Rural & $\begin{array}{l}239 \\
(22.4 \%)\end{array}$ & $830(77.6 \%)$ & $\begin{array}{l}0.91(0.66 \\
1.24)\end{array}$ & $0.79(0.48,1.31)$ \\
\hline \multicolumn{5}{|l|}{ Type of birth } \\
\hline Single birth & $\begin{array}{l}300 \\
(22.8 \%)\end{array}$ & $\begin{array}{l}1015 \\
(77.2 \%)\end{array}$ & 1 & 1 \\
\hline Multiple birth & $4(16.7 \%)$ & $20(83.3 \%)$ & $\begin{array}{l}0.68(0.23 \\
1.99)\end{array}$ & $0.61(0.17,2.15)$ \\
\hline \multicolumn{5}{|l|}{ Mothers` BMI } \\
\hline Over weight & $47(18.7 \%)$ & $205(81.3 \%)$ & 1 & 1 \\
\hline Normal weight & $\begin{array}{l}168 \\
(22.2 \%)\end{array}$ & $589(77.8 \%)$ & $\begin{array}{l}1.24(0.87 \\
1.78)\end{array}$ & $1.38(0.91,2.11)$ \\
\hline Under weight & 89 (27.0\%) & $241(73.0 \%)$ & $\begin{array}{l}1.61(1.08 \\
2.40)\end{array}$ & $1.64(1.03,2.64)^{\star}$ \\
\hline \multicolumn{5}{|c|}{ Size of child at birth } \\
\hline Large & $90(20.8 \%)$ & $343(79.2 \%)$ & 1 & 1 \\
\hline
\end{tabular}




\begin{tabular}{|c|c|c|c|c|}
\hline Variables & & Wasting & $\operatorname{COR}(95 \% \mathrm{Cl})$ & AOR (95\% Cl) \\
\hline Medium & $\begin{array}{l}130 \\
(22.5 \%)\end{array}$ & 447 (77.5\%) & $\begin{array}{l}1.11(0.82 \\
1.50)\end{array}$ & $1.19(0.84,1.67)$ \\
\hline Small & $84(25.5 \%)$ & $245(74.5 \%)$ & $\begin{array}{l}1.13(0.93 \\
1.84)\end{array}$ & $1.58(1.07,2.31)$ * \\
\hline \multicolumn{5}{|c|}{ Present of toilet facility } \\
\hline Yes & $\begin{array}{l}191 \\
(22.4 \%)\end{array}$ & $663(77.6 \%)$ & 1 & 1 \\
\hline No & $\begin{array}{l}113 \\
(23.3 \%)\end{array}$ & $372(76.7 \%)$ & $\begin{array}{l}1.05(0.81 \\
1.37)\end{array}$ & $1.06(0.74,1.53)$ \\
\hline \multicolumn{5}{|c|}{ Sex of household head } \\
\hline Male & $\begin{array}{l}211 \\
(23.3 \%)\end{array}$ & $696(76.7 \%)$ & 1 & 1 \\
\hline Female & $93(21.5 \%)$ & $339(78.5 \%)$ & $\begin{array}{l}0.91(0.69 \\
1.19)\end{array}$ & $0.86(0.63,2.16)$ \\
\hline \multicolumn{5}{|c|}{$\begin{array}{l}\text { Household wealth index } \\
\text { combined }\end{array}$} \\
\hline Poor & $\begin{array}{l}229 \\
(22.6 \%)\end{array}$ & $786(77.4 \%)$ & 1 & 1 \\
\hline Medium & $20(25.6 \%)$ & $58(74.4 \%)$ & $\begin{array}{l}1.18(0.70 \\
2.01)\end{array}$ & $1.17(0.63,2.16)$ \\
\hline Rich & $55(22.4 \%)$ & $191(77.6 \%)$ & $\begin{array}{l}0.99(0.71 \\
1.38)\end{array}$ & 0.93() $0.51,1.69$ \\
\hline \multicolumn{5}{|c|}{ Number of living children } \\
\hline $1-2$ & $60(21.4 \%)$ & $220(78.6 \%)$ & 1 & 1 \\
\hline $3-4$ & $93(22.9 \%)$ & $314(77.1 \%)$ & $\begin{array}{l}1.09(0.75 \\
1.57)\end{array}$ & $1.02(0.66,1.58)$ \\
\hline$>4$ & $\begin{array}{l}151 \\
(23.2 \%)\end{array}$ & $501(76.8 \%)$ & $\begin{array}{l}1.11(0.79 \\
1.55)\end{array}$ & $102(0.68,1.55)$ \\
\hline \multicolumn{5}{|c|}{ Sources of drinking water } \\
\hline Improved & $67(27.7 \%)$ & $175(72.3 \%)$ & 1 & 1 \\
\hline Unimproved & $\begin{array}{l}237 \\
(21.6 \%)\end{array}$ & $860(78.4 \%)$ & $\begin{array}{l}1.72(1.52, \\
2.99)\end{array}$ & $1.66(1.44,3.98) *$ \\
\hline \multicolumn{5}{|l|}{ Anemia level } \\
\hline
\end{tabular}




\begin{tabular}{|lllll|}
\hline Variables & & Wasting & COR (95\% Cl) & AOR (95\% Cl) \\
\hline Non-anemic & $44(23.9 \%)$ & $140(76.1 \%)$ & 1 & 1 \\
\hline Anemic & 209 & $626(075.0 \%$ & $1.06(0.73$, & $1.09(0.73,1.61)$ \\
& $(25.0 \%)$ & & $1.54)$ & \\
\hline
\end{tabular}

AOR: adjusted odds ratio; COR: crude odds ratio; $\mathrm{Cl}$ : confidence interval; * significant $\mathrm{p}$-value $<0.05$; ** significant $p$-value $<0.01$; significant $p$-value $<0.001,1=$ reference

\section{Discussion}

In the present study, the prevalence of stunting, underweight and wasting were $27.4 \%, 28.7 \%$ and $22.7 \%$, respectively. In this study, stunting is lower than the studies conducted in in Ethiopia (38.3\%) [25], in Shabelle zone, Somali region (30.4\%) [31], in Shinille District (33.4\%) [32], in Tigray region 39.1\% [5] and Takusa district $36.5 \%$ [33]. The prevalence of underweight in this finding is higher the studies conducted in Ethiopia (23.3\%) [25], in Tigray (23.9\%) [5], Dale district19\% [34] and Takusa district 19.5\% [33]. This figure is lower than the previously reported stunting (46.9\%) and underweight (33\%), but higher than wasting (11.6\%), and in Tigray region [17]. A similar study conducted in Nairobi Peri-Urban Slum reported a higher prevalence of stunting (30.2\%) but lower underweight (14.9\%) [35]. Similar studies conducted in Ethiopia reported a higher prevalence of stunting (46.3\%), the comparable prevalence of underweight (28.4\%), but lower prevalence of wasting (9.8\%) [36]. This might be due to the fact that there is a difference in barriers to under-nutrition such as cultural difference and other socio-demographic characteristics.

The prevalence of wasting in this study is higher compared to the study conducted in Ethiopia 10.1\% [25], in Haramaya district 10.7\% [37], in Dale district 14\% (32), in Tigray region [5] in Pakistan 10.7\% [38] and Nairobi Peri-Urban slum 4.5\% [35]. This divergence might be due to the difference in socioeconomic background, variation in sample size, dietary habits and type of meals among the study population. Similar studies conducted in Hidabu Abote District in the Oromia region reported a higher prevalence of stunting (47.6\%) and underweight (30.9\%), but a lower prevalence of wasting (16.7\%) [39]. A study conducted in rural Ethiopia also reported a higher finding $41.2 \%$ of stunting, but a lower finding $27 \%$ of underweight [40], whereas a study conducted in Nigeria reported a higher prevalence of stunting $47.6 \%$ but a lower prevalence of underweight 25.6\% [41]. While a study in the Bure Town of West Gojjam Zone (Amhara region) [42] reported a lower prevalence of stunting, underweight and wasting $(24.9 \%, 14.30 \%$ and $11.1 \%$, respectively). A similar study in east Gojjam Zone [43] reported lower prevalence of underweight $(15.3 \%)$ and wasting $(10 \%)$, but higher stunting $(44.7 \%)$. This could be due to there is a difference in obstacles to under-nutrition such as cultural differences and other socio-demographic characteristics.

Almost $16.1 \%$ of children were both stunted and underweight; the proportion of underweight and wasting was $11.7 \%$, the proportion of stunted and wasted was $5.5 \%$, and all three malnutrition conditions were only $4.7 \%$ children. The prevalence of both underweight and wasting at this finding is higher than the 
study finding in Amhara region (7.3\%) [44]. The prevalence of both stunted and underweight at this study is lower than compared to the study conducted in Ethiopia 19.47\% [25] and Amhara region (23.1\%) [44], but much higher than the study conducted in Kilimanjaro Region, Tanzania 33\% [45]. The prevalence of all the three conditions at this finding is lower than the study conducted in Amhara region (4.5\%) [44], in Kilimanjaro Region, Tanzania 12\% [45], but higher than in Ethiopia 3.87\% [25]. The variation might be due to socioeconomic background, geographical characteristics of the study area, access to health care, cultural difference in dietary habits and care practices.

According to the multivariable logistic regression analysis, the age of a child, type of birth, toilet facility and anemia level was significantly associated with being stunted. The risk of being stunted was 1.94 and 1.66 times more likely among children that were aged 25-47 and 48-59 as compared to those aged 024 months, respectively. This finding is in line with the studies conducted in Ethiopia [25], in Haramaya district [37], in Pakistan [38], in Amhara region [44] and in Kilimanjaro Region, Tanzania [45], but against the study conducted in Tigray region [5]. This might be because as children's' growth older, they may have fewer access attentions and not provide sufficient food from their families. Male children were 0.23 times less likely to be stunted as compared to female children. This result is in agreement with the study conducted in Bule Hora district, South Ethiopia [46], but in contradiction the studies conducted in Tigray region [5] and Pakistan [38]. The odds of stunting among children who were born in multi birth types were 2.86 times higher as compared to those born in single birth type. This finding is consistent with the study carried out in Tigray region [5]. The reason behind might be in multi birth type, there could be food competition between children and it leads to malnutrition; and the mothers' breasts may not produce enough milk for both children.

Children from the household who had not toilet facility were 1.45 times more likely to be stunted as compared to the children household who had a toilet facility. This result is in contradiction with the studies conducted in Tigray region [5] and in Bule Hora district, South Ethiopia [46]. This might be since lack of toilet facility is the main cause for intestinal parasites and microorganisms which leads to loss of appetite leading to poor nutritional status; this might repeated infection causes depressed immunity and making the severity and duration of disease more sever contributing to the poor nutritional status of the children. Children born from the medium and rich households were 0.44 and 0.22 times less likely to develop stunting as compared to those born from poor households. This fining supported with the literature reported that the poor wealth index is strongly correlated with under-five stunting [5, 42, 47-49]. The possible explanation for this might be mothers from households having the rich or middle wealth status were more likely to provide micronutrients in reached foods and seek medical treatment for their children. Being stunted of anemic children was 2.36 times more likely to be stunted as compared to nonanemic children. This finding has supported the study conducted in Ethiopia [25]. This is because the anemia causes the children to reduce feed intake and leads to malnutrition.

The children who were 25-47 and 48-59 aged groups were 1.67 and 1.70 times more likely to develop underweight as compared to those who were $0-24$ aged groups. This finding is supported by the study conducted in Ethiopia [25], but in contradiction the study conducted in Tigray region [5] and Pakistan [38]. 
This might be due to the fact that as children's' growth older, they may have less access to attentions and not provide sufficient food from their families. Compared to children large size at birth, the odds of underweight among children in the medium and small size at birth were 1.17 and 1.55 times higher. This study is in line with the study conducted in Tigray region [5] and in Pakistan [38]. The odds of being wasting were 0.11 times lower among female children than male children. Our results showed that male children were more likely to be stunted as compared to female children. The finding is consistent with the previous research reported that male children are more vulnerable to develop malnutrition because they require comparatively more calories for growth and development [50]. One of the reasons for low caloric intake in children is their low socioeconomic status as observed in our study. The anemic children were 1.65 times more likely to be underweight as compared to non-anemic children.

The results of the adjusted showed that male children were 0.35 times less likely to be wasting compared to female children. This finding is consistent with finding in Tigray region [5], but against in previous studies indicated that boys had a significantly worse nutritional status than girls [5]. The risk of being wasting among children who were from underweight and normal-weight mothers were 1.64 and 1.38 times higher compared to those born from overweight mothers. This study is in line with findings in Pakistan [38], in Ethiopia [25], in India [51] and Vietnam [52]. This finding is also similar to other previously conducted studies $[37,53]$. The children who had small size at birth were 1.58 times more likely to be wasting compared to those who had large size at birth. This result is supported by the study conducted in Tigray region [5]. Children whose household used unimproved water were 1.66 times more likely to be wasting as compared to the children household used improved water. This finding is supported with finding in Haramaya district, Eastern Ethiopia [37]. This might be because impure water is a vehicle for intestinal parasites which leads to loss of appetite leading to poor nutritional status; this might repeated infection causes depressed immunity and making the severity and duration of disease more sever contributing to the poor nutritional status of the children.

\section{Conclusion}

This study revealed individual- and community-level factors determined childhood malnutrition in Somali region children. Among the factors considered in this study, age of the child in months, type of birth, anemia level, size of child at birth, sex of a child, mothers' BMI and sources of drinking water were significantly associated with stunting, underweight and wasting. The authors concluded that undernutrition among under-five children was one of the public health problems in the study area. Thus, interventions should be focused on 25-59 months age of children, multiple birth type, anemic children, and small size of child at birth, female children, underweight mothers and improving access to improved drinking water to get better health care, to enhance the child's nutritional status, and reduce child mortality more rapidly.

\section{Limitations Of The Study}


A limitation was the use of a cross-sectional study design which could only generate a hypothesis regarding the role of independent variables on the nutritional status of children but not their cause and effect relationships.

\section{Abbreviations}

AOR

adjusted odds ratio; BMl:body mass index; COR:crude odds ratio; CSA:Central Statistical Agency; SPSS:Statistical Package for Social Science; DHS:Demographic and Health Surveys; EDHS:Ethiopian Demographic and Health Survey.

\section{Declarations}

\section{Ethics approval and consent to participate}

Ethics approval and consent to participate The EDHS 2016 has taken into account the standard ethical guidelines of the measure DHS program. The authors have obtained the data from measure DHS website (https://www.dhsprogram.com/data/dataset_admin/index.cfm) following their data obtaining procedure. The formal ethical clearance was obtained from the Demographic and Health Surveys (DHS) program.

\section{Consent for Publication}

Not applicable.

\section{Availability of data and material}

The data set used and analyzed during the current study is available from the corresponding author on reasonable request (in SPSS code).

\section{Competing interests}

The authors declare that they have no competing interests.

\section{Funding}

No funding was obtained for this study.

\section{Authors' contributions}

DK conceived the idea, drafts the manuscript and interpreted the results. DK, YM, HA and EW performed statistical analysis and help in results interpretation and writing. DK, YM, HA and EW critically reviewed 


\section{Acknowledgments}

The authors would like to thank Ethiopia Central Statistical Agency for permitting us to use the data for our study.

\section{References}

1. Hawkes C, Demaio AR, Branca F. Double-duty actions for ending malnutrition within a decade. The Lancet Global Health. 2017;5(8):e745-6.

2. Collins $\mathrm{S}$, et al. Management of severe acute malnutrition in children. The lancet. 2006;368(9551):1992-2000.

3. Okely AD, et al. Physical activity, sedentary behaviour, and sleep: movement behaviours in early life. The Lancet Child Adolescent Health. 2018;2(4):233-5.

4. Stoianova V, Private funding for humanitarian assistance. Producido por Global Humanitarian Assistance. 2013.

5. Woldeamanuel BT, Tesfaye TT, Risk factors associated with under-five stunting, wasting, and underweight based on Ethiopian Demographic Health Survey datasets in Tigray region, ethiopia. Journal of Nutrition and Metabolism. 2019; 2019.

6. Akombi BJ, et al. Child malnutrition in sub-Saharan Africa: A meta-analysis of demographic and health surveys (2006-2016). PloS one. 2017;12(5):e0177338.

7. Imdad A, Yakoob MY, Bhutta ZA. Impact of maternal education about complementary feeding and provision of complementary foods on child growth in developing countries. BMC Public Health. 2011;11(S3):S25.

8. Demographic E. Health survey central statistical agency Addis Ababa. Maryland: Ethiopia ICF International Calverton; 2011. pp. 180-6.

9. Alim F, Jahan F. Assessment of nutritional status of rural Anganwadi children of Aligarh under the ICDS (integrated child development services) and rural health. Studies on Home Community Science. 2012;6(2):95-8.

10. GotFDR E. National Nutrition Programme June 2013-June 2015. Addis Ababa: Government of Federal Democratic Republic of Ethiopia; 2013.

11. Windsma M, et al., Emergency obstetric care provision in southern Ethiopia: a facility-based survey. BMJ open. 2017; 7(11).

12. Benson T, et al. An assessment of the causes of malnutrition in Ethiopia: A contribution to the formulation of a National Nutrition Strategy for Ethiopia. Washington, DC, USA: International Food Policy Research Institute; 2005. 
13. Nabag FO. Comparative study of nutritional status of urban and rural school girl's children Khartoum state, Sudan. Journal of Science Technology. 2011;12(02):60-8.

14. Olack $B$, et al. Nutritional status of under-five children living in an informal urban settlement in Nairobi, Kenya. J Health Popul Nutr. 2011;29(4):357.

15. Rawe K, A life free from hunger: tackling child malnutrition. 2012. Save the Children.

16. El-Ghannam AR. The global problems of child malnutrition and mortality in different world regions. Journal of health social policy. 2003;16(4):1-26.

17. Mulugeta A, et al. Child malnutrition in Tigray, northern Ethiopia. East Afr Med J. 2010;87(6):248-54.

18. Edris M. Assessment of nutritional status of preschool children of Gumbrit, North West Ethiopia. Ethiopian Journal of Health Development. 2007;21(2):125-9.

19. Wollo E. Risk factors for child under-nutrition with a human rights edge in rural villages of North Wollo, Ethiopia. East Afr Med J. 2005;82(12):625-30.

20. Tarekegn SM, Lieberman LS, Giedraitis V. Determinants of maternal health service utilization in Ethiopia: analysis of the 2011 Ethiopian Demographic and Health Survey. BMC Pregnancy Childbirth. 2014;14(1):161.

21. Muchie KF. Quality of antenatal care services and completion of four or more antenatal care visits in Ethiopia: a finding based on a demographic and health survey. BMC Pregnancy Childbirth. 2017;17(1):1-7.

22. Wolde HF, et al. Factors affecting neonatal mortality in the general population: evidence from the 2016 Ethiopian Demographic and Health Survey (EDHS) - multilevel analysis. BMC Res Notes. 2019;12(1):610.

23. Population EOot, Commission HC, Summary and statistical report of the 2007 population and housing census: population size by age and sex. 2008. Federal Democratic Republic of Ethiopia, Population Census Commission.

24. Demographic CE. Health Survey-2011. Central Statistical Agency Addis Ababa. Ethiopia ICF International Calverton, Maryland, USA. 2012; 2016.

25. Tekile AK, Woya AA, Basha GW. Prevalence of malnutrition and associated factors among under-five children in Ethiopia: evidence from the 2016 Ethiopia Demographic and Health Survey. BMC Res Notes. 2019;12(1):391.

26. Tariq J, et al. Factors associated with undernutrition in children under the age of two years: secondary data analysis based on the Pakistan demographic and health survey 2012-2013. Nutrients. 2018;10(6):676.

27. Talukder A. Factors associated with malnutrition among under-five children: illustration using Bangladesh demographic and health survey, 2014 data. Children. 2017;4(10):88.

28. Asim M, Nawaz Y. Child malnutrition in Pakistan: evidence from literature. Children. 2018;5(5):60.

29. Das S, Gulshan J. Different forms of malnutrition among under five children in Bangladesh: a cross sectional study on prevalence and determinants. BMC Nutrition. 2017;3(1):1. 
30. Akombi BJ, et al. Multilevel analysis of factors associated with wasting and underweight among children under-five years in Nigeria. Nutrients. 2017;9(1):44.

31. GULED RA, et al. UNDERNUTRITION PREVALENCE AND ITS DETERMINANTS AMONG CHILDREN BELOW FIVE YEARS OF AGE IN SHABELLE ZONE, SOMALI REGION, EASTERN ETHIOPIA. INTERNATIONAL JOURNAL OF ALLIED HEALTH SCIENCES. 2017;1(2):72-91.

32. Ma'alin A, et al. Magnitude and factors associated with malnutrition in children 6-59 months of age in Shinille Woreda, Ethiopian Somali regional state: a cross-sectional study. BMC Nutrition. 2016;2(1):1-12.

33. Nigatu G, et al. Prevalence and associated factors of underweight among children 6-59 months of age in Takusa district, Northwest Ethiopia. Int J Equity Health. 2018;17(1):106.

34. Wolde $\mathrm{M}$, Berhan $\mathrm{Y}$, Chala A. Determinants of underweight, stunting and wasting among schoolchildren. BMC Public Health. 2015;15(1):8.

35. Chesire E, et al. Determinants of under nutrition among school age children in a Nairobi peri-urban slum. East Afr Med J. 2008;85(10):471-9.

36. CSACE I. Ethiopia demographic and health survey 2016. Maryland: CSA and ICF.: Addis Ababa, Ethiopia, and Rockville; 2016.

37. Yisak H, Gobena T, Mesfin F. Prevalence and risk factors for under nutrition among children under five at Haramaya district, Eastern Ethiopia. BMC Pediatr. 2015;15(1):212.

38. Khan S, Zaheer S, Safdar NF. Determinants of stunting, underweight and wasting among children $<5$ years of age: evidence from 2012-2013 Pakistan demographic and health survey. BMC Public Health. 2019;19(1):358.

39. Mengistu K, Alemu K, Destaw B. Prevalence of malnutrition and associated factors among children aged 6-59 months at Hidabu Abote District, North Shewa, Oromia Regional State. J nutr disorders ther. 2013;1:1-15.

40. Endris N, Asefa H, Dube L, Prevalence of malnutrition and associated factors among children in rural Ethiopia. BioMed research international. 2017.

41. Emmanuel A, et al., Malnutrition and associated factors among underfive in a Nigeria local government area. 2016.

42. Amare D, et al., Prevalence of undernutrition and its associated factors among children below five years of age in Bure Town, West Gojjam Zone, Amhara National Regional State, Northwest Ethiopia. Advances in Public Health. 2016.

43. Zeray A, Kibret GD, Leshargie CT. Prevalence and associated factors of undernutrition among underfive children from model and non-model households in east Gojjam zone, Northwest Ethiopia: a comparative cross-sectional study. BMC nutrition. 2019;5(1):27.

44. Damitie K, Mengesha YM. Prevalence of malnutrition and associated risk factors among under-five children in Amhara Region, Ethiopia: evidence from 2016 Ethiopian Demographic and Health Survey. unpublished. 2020. 
45. Mgongo $\mathrm{M}$, et al. Underweight, stunting and wasting among children in Kilimanjaro Region, Tanzania; a population-based cross-sectional study. Int J Environ Res Public Health. 2017;14(5):509.

46. Asfaw $\mathrm{M}$, et al. Prevalence of undernutrition and associated factors among children aged between six to fifty nine months in Bule Hora district, South Ethiopia. BMC Public health. 2015;15(1):41.

47. Amare $\mathrm{M}$, et al., Study of the determinants of chronic malnutrition in Northern Nigeria: quantitative evidence from the Nigeria demographic and health surveys international food policy research institute (IFPRI) working paper 45 (September 2017). Food and nutrition bulletin. 2018; 39(2): p. 296-314.

48. Awoke A, Ayana M, Gualu T. Determinants of severe acute malnutrition among under five children in rural Enebsie Sarmidr District, East Gojjam Zone, North West Ethiopia, 2016. BMC nutrition. 2018;4(1):4.

49. Debeko DD, Goshu AT. Nutritional status of under-five children in Hawassa Zuria District, Southern Ethiopia. Am J Health Res. 2015;3(5):286-92.

50. Demissie S, Worku A. Magnitude and factors associated with malnutrition in children 6-59 months of age in pastoral community of Dollo Ado district, Somali region, Ethiopia. Sci J Public Health. 2013;1(4):175-83.

51. Kumar D, et al. Influence of infant-feeding practices on nutritional status of under-five children. The Indian Journal of Pediatrics. 2006;73(5):417-21.

52. Hien NN, Hoa NN. Under three years of age in nghean, Vietnam. Pakistan Journal of Nutrition. 2009;8(7):958-64.

53. Siddiqi MNA, Haque MN, Goni MA. Malnutrition of under-five children: evidence from Bangladesh. Asian Journal of medical sciences. 2011;2(2):113-9.

\section{Supplementary Files}

This is a list of supplementary files associated with this preprint. Click to download.

- DHSLetter.pdf 